\title{
A Random Change Point Model for Cognitive Decline in Alzheimer's Disease and Mild Cognitive Impairment
}

\author{
Lei Yu ${ }^{a, b}$ Patricia Boyle ${ }^{a, c}$ Robert S. Wilson ${ }^{a-c}$ Eisuke Segawa ${ }^{a}$ \\ Sue Leurgans ${ }^{a, b}$, Philip L. De Jager ${ }^{\mathrm{e}-\mathrm{g}}$ David A. Bennett ${ }^{\mathrm{a}, \mathrm{b}}$ \\ ${ }^{a}$ Rush Alzheimer's Disease Center and Departments of ${ }^{b}$ Neurological Sciences, ${ }^{c}$ Behavioral Sciences and \\ dPreventive Medicine, Rush University Medical Center, Chicago, Ill., e Program in Translational NeuroPsychiatric \\ Genomics, Institute for the Neurosciences, Departments of Neurology and Psychiatry, Brigham and Women's \\ Hospital, and ${ }^{\mathrm{f} H a r v a r d}$ Medical School, Boston, Mass., and 9 Program in Medical and Population Genetics, \\ Broad Institute, Cambridge, Mass., USA
}

\section{Key Words \\ Change point model $\cdot$ Cognitive decline $\cdot$ Alzheimer's \\ disease $\cdot$ Amnestic mild cognitive impairment}

\begin{abstract}
Background: We present a random change point model to characterize decline in cognition among community-based elderly who developed Alzheimer's disease (AD) or amnestic mild cognitive impairment $(\mathrm{MCl})$, and to examine how decline varies with age, sex, education, and APOE status. Methods: Using longitudinal cohort data on cognitive function, we fit a piecewise linear trajectory with a random change point that allows different rates of cognitive decline before and after the change point. We estimated the change point that signals the onset of cognitive impairment, and examined the association of risk factors with the location of the change point as well as the rates of decline before and after the change point. Results: Among participants who were dementia free at enrollment and developed incident $A D$, the change point occurred on average 5.7 years after enrollment
\end{abstract}

and the rate of cognitive decline after the change point nearly quadrupled. Age, education, and APOE status play important but different roles in the timing of the onset of cognitive impairment and in the rates of decline before and after its onset. Results were similar among participants who were cognitively unimpaired at enrollment but later developed amnestic $\mathrm{MCl}$ or AD. Conclusions: The random change point model provides a more comprehensive understanding of the relation of risk factors with the onset of cognitive impairment and rates of decline before and after its onset.

Copyright $\odot 2012$ S. Karger AG, Basel

\section{Introduction}

Alzheimer's disease (AD) is the most common cause of dementia among the elderly. Prevention and delay of disease onset serve as the best long-term strategies and would have a substantial public health impact $[1,2]$. Recent data show that the onset of cognitive decline can occur months or even years before a clinical diagnosis of

\section{KARGER}

Fax +4161306 1234

E-Mail karger@karger.ch

www.karger.com
(C) 2012 S. Karger AG, Basel

$0251-5350 / 12 / 0392-0073 \$ 38.00 / 0$

Accessible online at:

www.karger.com/ned
Lei $\mathrm{Yu}, \mathrm{PhD}$

Rush Alzheimer's Disease Center, Armour Academic Facility

600 South Paulina Street, Suite \#1020B

Chicago, IL 60612 (USA)

Tel. +1 312942 0543,E-Mail lei_yu@rush.edu 
AD is made [3-5]. Further, clinical-pathologic studies find that $\mathrm{AD}$ and other neuropathologies are common among persons without dementia and related to mild cognitive impairment (MCI) and level of cognitive function even among persons without MCI [6-8]. These findings have led to a new conceptualization of $\mathrm{AD}$ as a continuum from an asymptomatic pathophysiologic process to $\mathrm{MCI}$ and finally clinical dementia due to $\mathrm{AD}$ [9-11]. Understanding the trajectories of cognition as persons cross this continuum and the relation of risk factors to these trajectories is needed in order to identify and evaluate strategies to reduce the large and rapidly increasing burden of cognitive decline on our aging population.

Prior literature suggests that risk factors associated with cognitive decline and the onset of $\mathrm{AD}$ dementia might differ from those related to cognitive decline following disease onset; in fact, some may even exhibit opposite effects before and after clinical diagnosis. For example, incidence of $\mathrm{AD}$ increases with age and the prevalence of $\mathrm{AD}$ doubles every 5 years after age 65 [12]. By contrast, age appears to be unrelated to rate of cognitive decline among community-based older persons with a clinical AD diagnosis [13], and in clinical settings a more rapid decline is found among persons who were younger at disease onset [14]. Some studies suggest that women have a higher incidence of $\mathrm{AD}$ [15], but no sex difference is found in decline of language in $\mathrm{AD}$ [16]. Persons with more years of formal education tend to have a reduced risk of incident $\mathrm{AD}$, but more education is associated with a faster rate of cognitive decline among persons with $\mathrm{AD}$ $[17,18]$. This finding has been supported by a cohort study that examines the relation of education to the trajectory of cognition prior to the clinical diagnosis of $\mathrm{AD}$, where persons with more education have a later onset of change point of cognition, but a more rapid decline after the change point [19]. It is also well known that the apolipoprotein $\varepsilon 4$ allele (APOE $\varepsilon 4$ ) is associated with a faster rate of cognitive decline [20] and an elevated risk of $\mathrm{AD}$ dementia [21]. By contrast, some studies suggest that the polymorphism is not related to the rate of decline after the $\mathrm{AD}$ diagnosis $[22,23]$. Together, these data suggest that linking risk factors to the trajectory of cognitive decline across the spectrum of $\mathrm{AD}$ could provide important insights into where risk factors are operative. This has critical implications for the timing of treatment and prevention.

To characterize trajectory of change in cognitive function, we present a random change point model that allows different rates of decline before and after the change point and estimate the location of the change point that signals the onset of cognitive impairment. This approach enables us to investigate, in a unified model, the association of multiple risk factors with the timing of the onset of cognitive impairment and with the rates of cognitive decline before and after the onset. Using data from nearly 400 community-based elderly who underwent detailed annual cognitive assessments for up to 18 years, we first examined participants who were free of dementia at enrollment and later developed AD. In subsequent analyses, we examined those who were cognitively unimpaired at enrollment and developed amnestic MCI or AD. We restricted the analyses to those participants who had at least 5 cognitive evaluations in order to better capture the trajectory of cognitive change and allow for stable estimates both before and after the change point.

\section{Subjects and Methods}

\section{Subjects}

Subjects are participants enrolled in two ongoing clinicalpathologic cohort studies of aging and dementia, the Religious Orders Studies (ROS) [24] and the Memory and Aging Project (MAP) [25]. Participants from ROS are older Catholic nuns, priests, and brothers who agreed to annual uniform structured clinical evaluations including a medical history, cognitive function testing, neuropsychological examination, blood specimen collection, and brain donation at time-of-death. They come from about 40 groups across the United States. Since January 1994, more than 1,100 persons completed the baseline clinical evaluation and the follow-up rate of survivors exceeds 95\%. Participants from MAP are residents of approximately 40 senior housing facilities in the Chicago metropolitan area. As in ROS, participants from MAP also have consented to undergo annual uniform structured clinical evaluations. Since October 1997, the MAP study has enrolled more than 1,400 participants with a follow-up rate of over $90 \%$ among the survivors. Both studies were approved by the Institutional Review Board of Rush University Medical Center.

\section{Genotyping of APOE}

Blood was collected with acid citrate dextrose anticoagulant, stored at room temperature, and underwent lymphocyte separation within $24 \mathrm{~h}$ of collection. DNA was extracted from approximately 2-3 million mononuclear cells. Genotyping was done at the Agencourt Bioscience Corporation (Beverly, Mass., USA), utilizing high-throughput sequencing of codon 112 (position 3937) and codon 158 (position 4075) of exon 4 of the APOE gene as previously described [26].

Participants with 1 or more copies of $\varepsilon 4$ allele (i.e. $\varepsilon 2 / 4, \varepsilon 3 / 4$, $\varepsilon 4 / 4)$ are considered $\varepsilon 4$ carriers. All other participants with other allele combinations (i.e. $\varepsilon 2 / 2, \varepsilon 2 / 3$, or $\varepsilon 3 / 3$ ) are considered noncarriers.

\section{Cognitive Function and Clinical Diagnosis}

Detailed annual cognitive function data has been collected from participants in the two cohorts. Briefly, cognitive function 
was assessed via a battery of 17 tests which were combined into a composite measure of cognition [27]. Higher scores indicate better performance in cognitive function. Psychometric information on these summary scores is reported in previous publications [28].

Clinical diagnoses were performed in three steps [29]. The steps consist of scoring of tests by computer, assignment of cognitive impairment by a neuropsychologist, and determination of dementia and $\mathrm{AD}$ by a clinician using standard criteria which require evidence of cognitive decline in memory and at least one other domain of cognitive function [30]. MCI was diagnosed in the presence of cognitive impairment as determined by the neuropsychologist and the absence of dementia as determined by the clinician [31]. Amnestic MCI required a diagnosis of MCI and impairment of episodic memory. The relations between clinical diagnoses of $\mathrm{AD}$ and of amnestic MCI with the pathologic diagnosis of $\mathrm{AD}$ have been previously reported [32].

\section{A Random Change Point Model}

We characterize the change in cognition by modeling a piecewise linear trajectory with an unknown change point. The model follows a hierarchical structure as below:

$\begin{aligned} y_{i j}= & \left(\theta_{i 0}+\gamma_{i 0}\right)+\left(\theta_{i 1}+\gamma_{i 1}\right)\left(T_{i j}, \theta_{i 3}+\gamma_{i 3}\right)_{\min }+\left(\theta_{i 1}+\gamma_{i 1}\right)\left(T_{i j}-\right. \\ & \left.\left(\theta_{i 3}+\gamma_{i 3}\right)\right)_{+}+e_{i j}\end{aligned}$

The outcome $y_{i j}$ is the longitudinal measure of cognitive function for subject $i$ at visit $j, i=1, \ldots, m$ and $j=1, \ldots, n_{i} . \theta_{i 0}+\gamma_{i 0}, \theta_{i 1}+\gamma_{i 1}$, $\theta_{i 2}+\gamma_{i 2}$ and $\theta_{i 3}+\gamma_{i 3}$ represent, respectively, the subject-specific intercept, the slope before and after the change point, and the change point. Here, fixed effects, $\left(\theta_{i 0}, \theta_{i 1}, \theta_{i 2}, \theta_{i 3}\right)^{\prime}$, are further parameterized as linear functions of terms for age at enrollment, sex, education and $A P O E$ status, $\theta_{i k}=\mathrm{X}_{i} \beta_{k}, k=0,1,2,3$. Random effects, $\left(\gamma_{i 0}, \gamma_{i 1}, \gamma_{i 2}, \gamma_{i 3}\right)^{\prime}$, assume multivariate normal with means $0 \mathrm{~s}$ and unknown variance covariance structure $\Sigma_{4 \times 4}$. $T_{i j}$ is time (in years since enrollment) for subject $i$ at visit $j$. In order to differentiate the rate of change in cognition before and after the change point (denoted by $\theta_{i 3}+\gamma_{i 3}$ ), we specify two separate column vectors of time in the model's design matrix. The first column vector is defined as the minimum of $\left(T_{i j}, \theta_{i 3}+\gamma_{i 3}\right)$ and the second column vector as $\left(T_{i j}-\left(\theta_{i 3}+\gamma_{i 3}\right)\right)_{+}$, where $\left(T_{i j}-\left(\theta_{i 3}+\gamma_{i 3}\right)\right)_{+}$takes value of zero if $T_{i j} \leq\left(\theta_{i 3}+\gamma_{i 3}\right)$ and $T_{i j}-\left(\theta_{i 3}+\gamma_{i 3}\right)$ if $T_{i j}>\left(\theta_{i 3}+\gamma_{i 3}\right)$. The residual error $e_{i j}$ assumes normal with mean 0 and unknown variance $\sigma^{2}$. Similar models have been applied previously in other contexts $[33,34]$.

The change point is intended to signal the onset of cognitive impairment. A unique feature of this model is that the change point is estimated empirically, rather than anchored at time of the clinical diagnosis. Clinical diagnosis was performed annually by clinicians blinded to all previously collected data, but clinical diagnoses can be imprecise. Dissociation of the change point from the clinical diagnosis allows us to directly examine the temporal relation of the change point to the clinical diagnosis. The model also allows us to investigate whether risk factors modify the location of the change point, as well as the slopes of decline before and after the change point.

In analyses, we first compared the location of the change point with the time of the clinical AD diagnosis among those who were dementia free at enrollment. To do so, we inspected the kernel density estimation, computed Pearson's correlation coefficient, and examined the distributional characteristics of individual dif-
Table 1. Demographic characteristics of the incident AD group $(\mathrm{n}=399)$
Age at enrollment, years

Age at $\mathrm{AD}$ diagnosis, years

Time to $\mathrm{AD}$, years

Education, years

Number of cognitive evaluations

Female

Carriers of $A P O E \varepsilon 4$ allele

Data presented as mean \pm SD or $n(\%)$.
$81.0 \pm 6.3$

$86.9 \pm 6.3$

$5.9 \pm 3.7$

$16.5 \pm 3.6$

$9.2 \pm 3.2$

$300(75.2)$

$118(29.6)$ ferences between the change point and the time of the clinical diagnosis. Next, we examined whether APOE status and demographic variables shifted the location of the change point and examined how these variables were associated with the rate of decline before and after the change point. Next, in view of the recent diagnostic guidelines for AD dementia, we extended the spectrum of disease by also including participants who developed amnestic MCI. To do so, we repeated the analyses by excluding from the previous sample participants who had MCI at enrollment, and adding those who were cognitively unimpaired at enrollment but developed amnestic MCI during the follow-up.

Parameters were estimated using a Bayesian Monte Carlo Markov Chain (MCMC) approach with conventional conjugate priors. Specifically, each coefficient for the fixed effects $\beta$ assumes normal prior with mean 0 and variance 10,000 ; inverse of variance covariance matrix for random effects assumes Wishart $(\mathrm{R}, 4)$, where $\mathrm{R}$ is a 4 by 4 diagonal matrix of 0.1 ; and inverse of residual variance assumes gamma with parameters of $0.001 \mathrm{~s}$. As sensitivity analyses, alternative choice of priors was used in modeling the data on incident $\mathrm{AD}$ group and the output was presented as online appendix (Appendix A), and the results suggest that estimation overall was robust to priors selection. Posterior means from the sampling chain were used as the point estimates and posterior 2.5 and 97.5 quantiles were used to construct $95 \%$ credible intervals. The analyses were implemented in OpenBUGS [35].

\section{Results}

\section{Demographics of the Incident AD Group}

At the time of analysis, 277 individuals from the ROS and 231 from the MAP had developed incident AD. Of those 508, 38 had missing data on $A P O E$, and 71 had fewer than 5 cognitive evaluations. This left 399 individuals eligible for the analysis (table 1). The mean (SD) age at enrollment was $81.0(6.3)$ years. The mean (SD) level of education was 16.5 (3.6) years, and 300 (75.2\%) of the participants were female. 118 (29.6\%) of the participants were APOE $\varepsilon 4$ carriers. Participants completed a mean (SD) of 9.2 (3.2) annual evaluations. 
Table 2. Association of age, sex, education and $A P O E$ with cognitive decline

\begin{tabular}{|c|c|c|c|c|}
\hline Effect & \multicolumn{2}{|l|}{ Model A } & \multicolumn{2}{|l|}{ Model B } \\
\hline Age & $-0.019 \pm 0.004$ & $-0.027,-0.011$ & $-0.011 \pm 0.003$ & $-0.017,-0.006$ \\
\hline Sex male & $-0.147 \pm 0.057$ & $-0.261,-0.037$ & $-0.069 \pm 0.042$ & $-0.152,0.013$ \\
\hline Education, years & $0.042 \pm 0.007$ & $0.028,0.056$ & $0.043 \pm 0.005$ & $0.033,0.053$ \\
\hline Age $\times$ Slope_before & $-0.001 \pm 0.001$ & $-0.003,0.0008$ & $-0.001 \pm 0.0007$ & $-0.003,-2.8 \mathrm{E}-6$ \\
\hline Sex $\times$ Slope & $0.006 \pm 0.014$ & $-0.020,0.032$ & $0.012 \pm 0.010$ & $-0.007,0.033$ \\
\hline Education, years $\times$ Slope before & $0.0003 \pm 0.002$ & $-0.003,0.004$ & $-0.002 \pm 0.001$ & $-0.004,0.001$ \\
\hline$A P O E \times$ Slope $\_$before & $-0.033 \pm 0.013$ & $-0.058,-0.008$ & $-0.024 \pm 0.010$ & $-0.043,-0.003$ \\
\hline Slope_after & $-0.337 \pm 0.023$ & $-0.383,-0.293$ & $-0.273 \pm 0.021$ & $-0.314,-0.233$ \\
\hline Age $\times$ Slope_after & $-0.003 \pm 0.003$ & $-0.008,0.002$ & $-0.006 \pm 0.026$ & $-0.011,-0.001$ \\
\hline Change point & $5.688 \pm 0.234$ & $5.228,6.142$ & $5.554 \pm 0.228$ & $5.107,6.002$ \\
\hline Age $\times$ change point & $-0.138 \pm 0.026$ & $-0.188,-0.088$ & $-0.137 \pm 0.028$ & $-0.190,-0.083$ \\
\hline Sex $\times$ change point & $-0.608 \pm 0.358$ & $-1.297,0.084$ & $-0.699 \pm 0.363$ & $-1.396,0.018$ \\
\hline Education, years $\times$ change point & $0.155 \pm 0.045$ & $0.066,0.244$ & $0.181 \pm 0.044$ & $0.095,0.268$ \\
\hline$A P O E \times$ change point & $-0.519 \pm 0.337$ & $-1.172,0.150$ & $-0.386 \pm 0.361$ & $-1.091,0.317$ \\
\hline \multicolumn{5}{|l|}{ SD of random effect } \\
\hline Random intercept & $0.435 \pm 0.129$ & $0.399,0.474$ & $0.324 \pm 0.098$ & $0.296,0.354$ \\
\hline Random Slope_before & $0.074 \pm 0.028$ & $0.064,0.085$ & $0.056 \pm 0.021$ & $0.048,0.063$ \\
\hline Random Slope_after & $0.247 \pm 0.092$ & $0.215,0.281$ & $0.247 \pm 0.091$ & $0.216,0.281$ \\
\hline Random change point & $2.218 \pm 0.716$ & $1.993,2.448$ & $2.203 \pm 0.726$ & $1.974,2.442$ \\
\hline
\end{tabular}

$\mathrm{CI}=$ Credible interval; Age $=$ age at enrollment centered at 79; Education, years = years of education centered at $16 ; A P O E=1$ for carriers of $\varepsilon 4$ allele, 0 for noncarriers; Slope_before = slope estimate before the change point; Slope_after = slope estimate after the change point.

\section{The Change Point in the Incident AD Group}

The posterior mean of the change point was 5.7 years $(\mathrm{SD}=0.23,95 \% \mathrm{CI}=[5.2,6.1])$ after enrollment (table 2, model A). On average, the change point occurred proximate to the first clinical AD diagnosis which had a mean of 5.9 years. Details on the comparison are illustrated in figure 1. Age at enrollment and years of education were both associated with the location of the change point. Older age at enrollment moved the change point earlier, while persons with more years of education had a later change point. Sex and $A P O E$ status were not related to the location of the change point.

Cognitive Decline before and after the Change Point in the Incident $A D$ Group

The estimated mean rate of change in cognition before the change point was -0.090 standard units per year $(\mathrm{SD}=0.008,95 \% \mathrm{CI}=[-0.106,-0.074])$, and the slope of decline after the change point was nearly four-fold faster, with a mean estimate of $-0.337(\mathrm{SD}=0.023,95 \% \mathrm{CI}=$ $[-0.383,-0.293])$. Age at enrollment was not associated with the slopes before or after the change point (fig. 2a). Education was not associated with the decline before the change point, but more years of education was associated with faster decline after the change point (fig. 2b). Sex was not related to either slope of decline. Carriers of the $\varepsilon 4$ allele exhibited faster decline in cognition both before and after the change point (fig. 2c). There was about a 37\% more rapid decline before and about a $28 \%$ more rapid decline after the change point among $\varepsilon 4$ carriers.

\section{Demographics of the Incident Amnestic MCI or AD Group}

Amnestic MCI is often an early manifestation of AD. Thus, in a separate set of analyses we included participants who developed either amnestic MCI or AD. There 


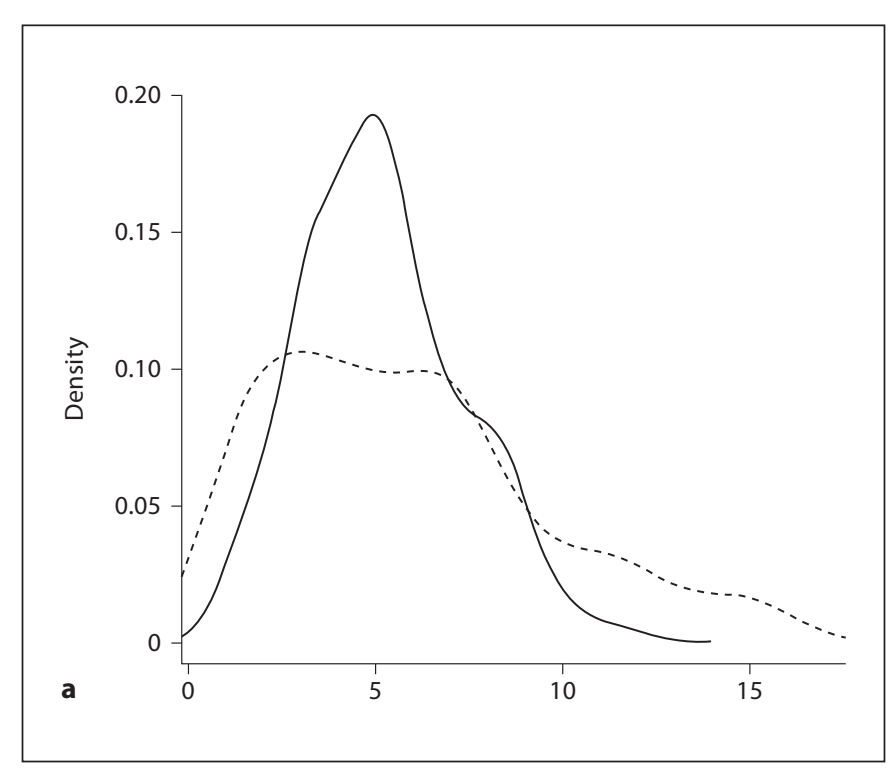

Fig. 1. Comparison of the change point with the clinical diagnosis (the incident AD group). a Posterior distribution of the change point. The solid curve is a kernel density estimate of the change point, in years since enrollment. The dashed curve is the density estimate of the first clinical AD diagnosis. b Scatter plot of time of clinical AD diagnosis versus the change point. On the horizontal axis is the estimated change point, in years since enrollment. On the vertical axis is the corresponding clinical AD diagnosis. The plot is overlaid with a 45 -degree reference line. c Histogram of difference between change point and $\mathrm{AD}$ diagnosis, overlaid with a normal density.

were 498 individuals from the two cohorts (249 in ROS, and 249 in MAP) who were cognitively unimpaired at enrollment but had either a clinical diagnosis of amnestic MCI or AD during the follow-up. Of those, 38 did not have data on $A P O E$, and 61 had fewer than 5 cognitive evaluations. This left 399 individuals eligible for this analysis (table 3). The mean (SD) age at enrollment was 79.4 (6.3) years. The mean (SD) level of education was 16.5 (3.8) years, and $284(71.2 \%)$ of the participants were female. 104 (26.1\%) of the participants were APOE $\varepsilon 4$ carriers. Participants completed a mean (SD) of 9.8 (3.5) annual evaluations. 123 participants were diagnosed with
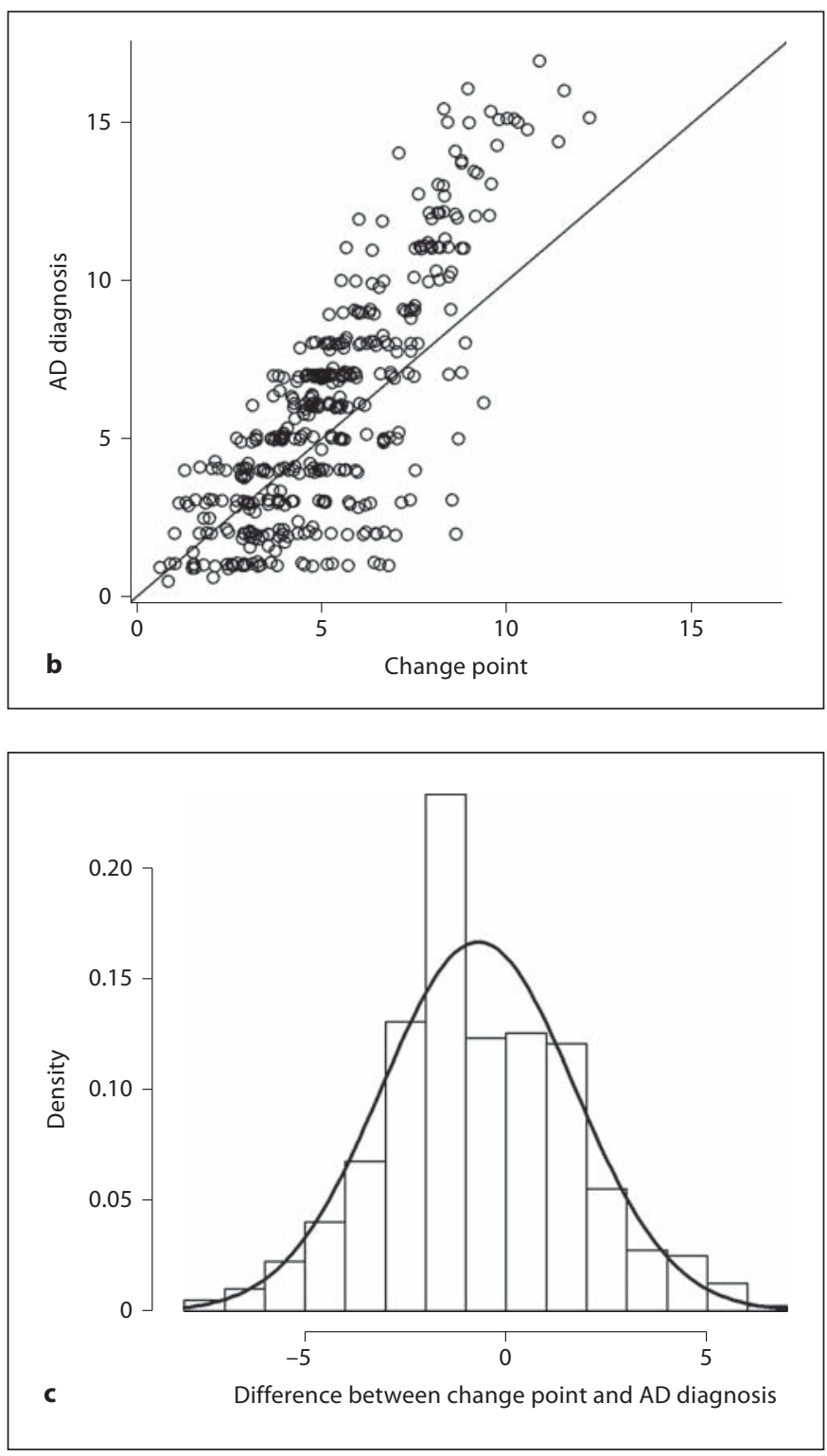

amnestic MCI and subsequently with $\mathrm{AD}, 185$ were only diagnosed with amnestic MCI and 91 were diagnosed with $\mathrm{AD}$ without a previous amnestic MCI diagnosis.

\section{The Change Point in the Incident MCI or AD Group}

Among participants who were cognitively unimpaired at the baseline but later developed amnestic MCI or $\mathrm{AD}$, the posterior mean of the change point was 5.6 years $(\mathrm{SD}=0.23,95 \% \mathrm{CI}=[5.1,6.0])$ after enrollment (table 2, model B). On average, the change point occurred after the clinical diagnosis of amnestic MCI which had a mean of 5.0 years. Details on the comparison are illustrated in fig- 


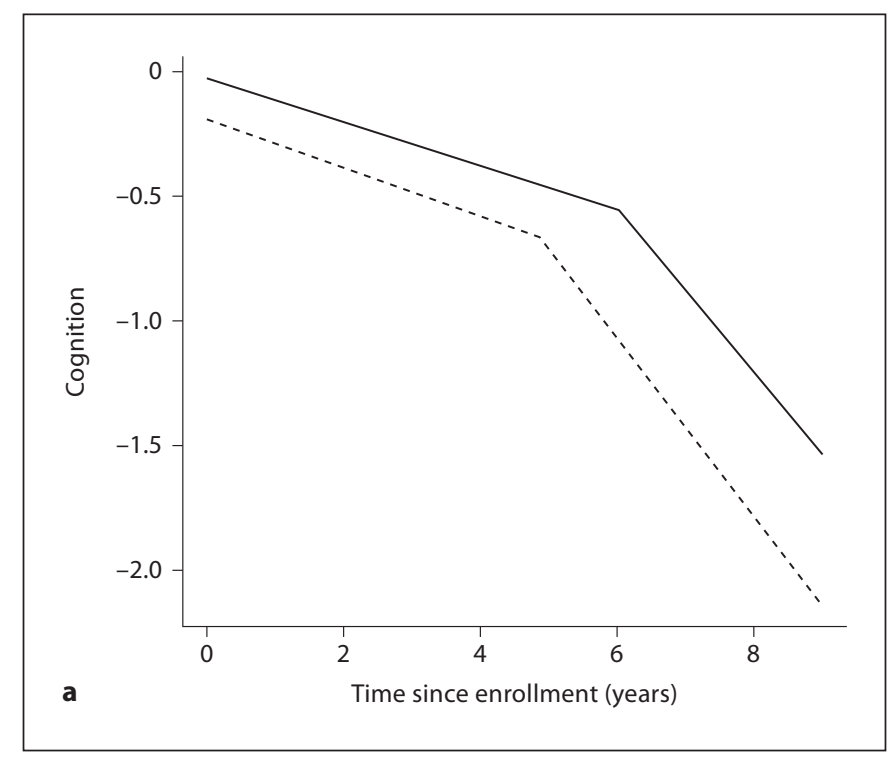

Fig. 2. Effect of age, education, and $A P O E \varepsilon 4$ allele on the decline in cognition (the incident AD group). a Effect of age. Estimated mean trajectory of cognitive decline over time for persons with younger versus older age at enrollment. The dotted line represents a typical subject with 75th percentile age at enrollment $(85.2$ years), and the solid line refers to a subject with 25 th percentile age at enrollment (76.5 years). b Effect of education. Estimated mean trajectories of cognitive decline over time for groups with lower versus higher level of education. The dotted line represents a typical subject with 75 th percentile education (19 years), and the solid line refers to a subject with 25th percentile education (14 years). c Effect of $A P O E \varepsilon 4$. Estimated mean trajectories of cognitive decline over time for groups with and without $\varepsilon 4$ allele. The dotted line represents a typical subject without $\varepsilon 4$ allele, and the solid line refers to the one with at least one $\varepsilon 4$ allele.

Table 3. Demographic characteristics of the incident amnestic MCI or AD group $(n=399)$

\begin{tabular}{lr}
\hline Age at enrollment, years & $79.4 \pm 6.3$ \\
Age at first amnestic MCI, years $(\mathrm{n}=308)$ & $84.5 \pm 6.2$ \\
Age at first AD, years $(\mathrm{n}=214)$ & $87.5 \pm 5.9$ \\
Time to amnestic MCI, years & $5.0 \pm 3.7$ \\
Time to AD, years & $7.4 \pm 3.5$ \\
Education, years & $16.5 \pm 3.8$ \\
Number of cognitive evaluations & $9.8 \pm 3.5$ \\
Female & $284(71.2)$ \\
Carriers of APOE $\varepsilon 4$ allele & $104(26.1)$
\end{tabular}

Data presented as mean $\pm \mathrm{SD}$ or $\mathrm{n}(\%)$
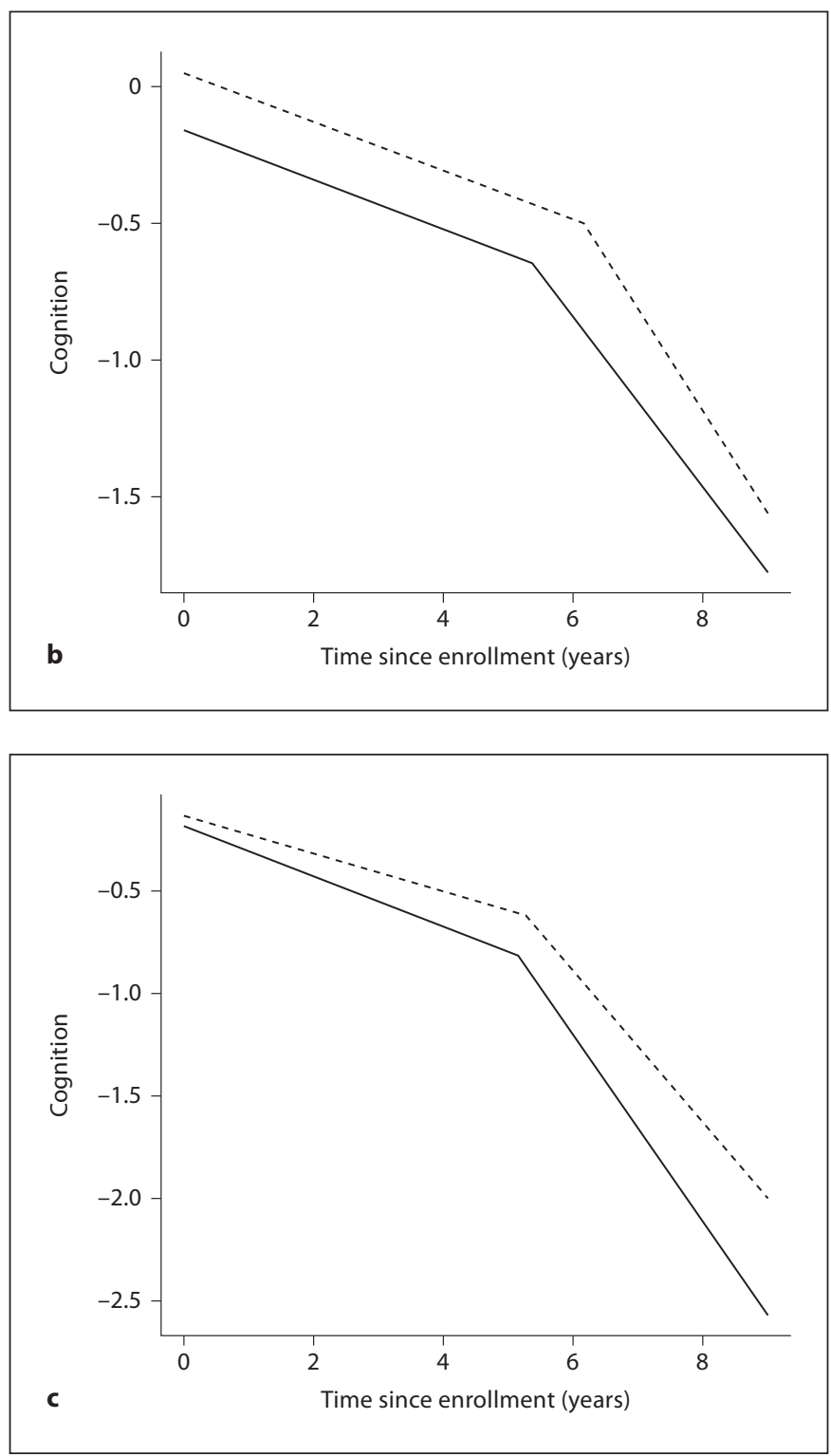

ure 3. Consistent with the previous findings, older age at enrollment was associated with an earlier change point, while more education delayed the change point. Again, sex and $A P O E$ status were not associated with the location of the change point.

Cognitive Decline before and after the Change Point in the Incident MCI or AD Group

Compared with the results from incident $\mathrm{AD}$ analysis, the estimated mean rate of decline in cognition prior to the change point was slower $(\mathrm{EST}=-0.05, \mathrm{SD}=0.007$, $95 \% \mathrm{CI}=[-0.06,-0.04])$ among persons who developed 

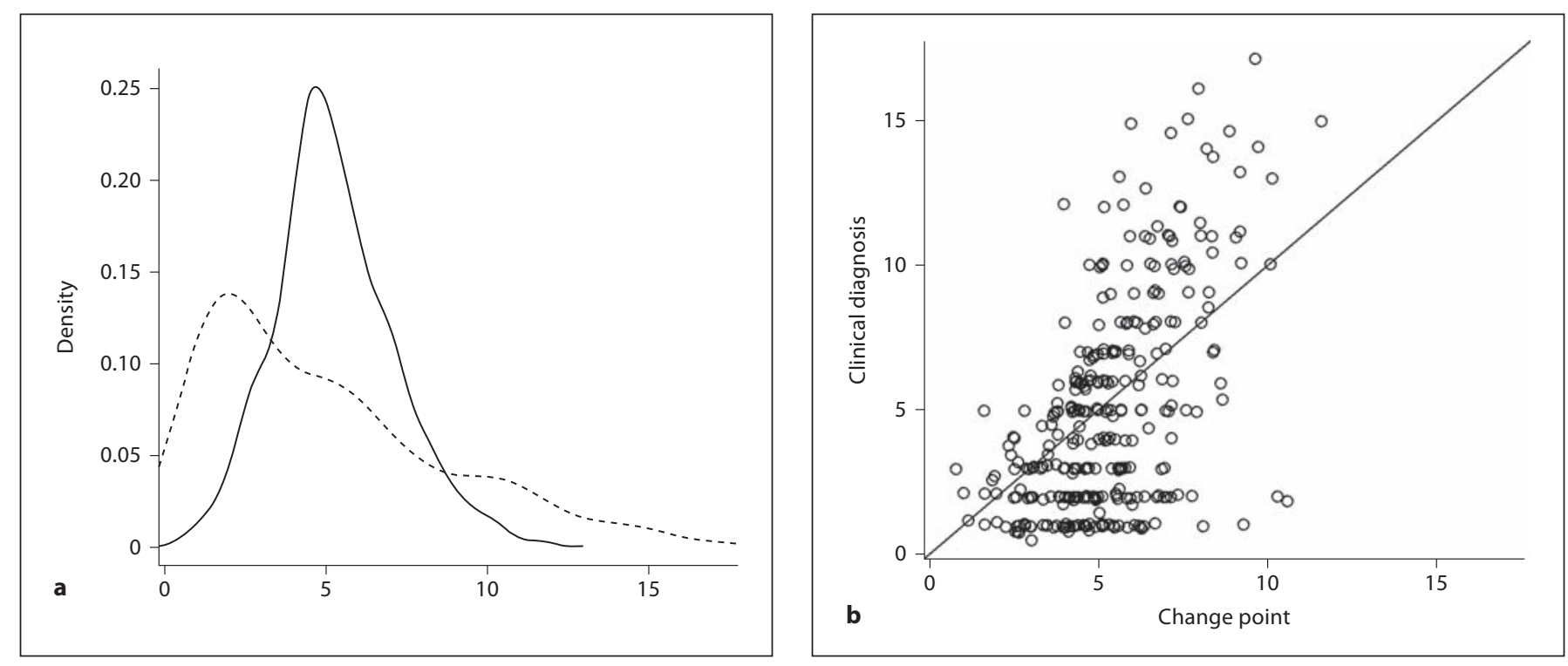

Fig. 3. Comparison of the change point with the clinical diagnoses (the incident amnestic MCI or AD group). a Posterior distribution of the change point. The solid curve is a kernel density estimate of the change point, in years since enrollment. The dashed curve is the density estimate of the first clinical diagnosis of amnestic MCI. b Scatter plot of time of clinical amnestic MCI diagnosis versus the change point. On the horizontal axis is the estimated change point, in years since enrollment. On the vertical axis is the corresponding clinical diagnosis of amnestic MCI. The plot is overlaid with a 45-degree reference line. c Histogram of difference between change point with clinical diagnosis, overlaid with a normal density.

incident $\mathrm{MCI}$ or $\mathrm{AD}$. The decline after the change point was about five times faster than that before the change point $(\mathrm{EST}=-0.27, \mathrm{SD}=0.021,95 \% \mathrm{CI}=[-0.31,-0.23])$. Age at enrollment was now associated with the rate of cognitive decline, such that the older the age at enrollment, the faster the decline in cognition before and after the change point (fig. 4a). More years of education was again associated with faster decline after the change point (fig. 4b). $\varepsilon 4$ carriers exhibited about a $47 \%$ faster rate of decline in cognition before and about a $38 \%$ faster rate of decline after the change point (fig. 4c).

\section{Discussion}

To date, the vast majority of studies on risk factors for cognitive decline have relied on linear models that assume a constant, straight-line decline. Here we present a random change point model that allows the rate of cognitive decline to vary at an empirically derived change point. The specified model serves as a convenient tool to characterize the nonlinear trajectories of cognitive decline among community-based elderly who developed incident AD or amnestic MCI. Further, the model allows for simultaneous evaluation of the effect of risk factors on 


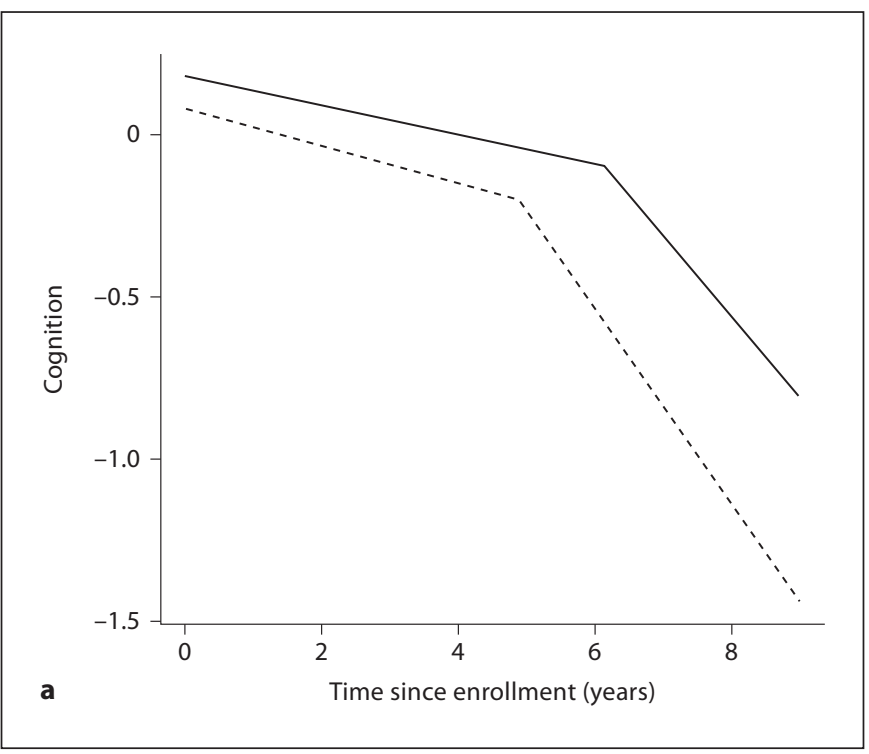

Fig. 4. Effect of age, education, and $A P O E \varepsilon 4$ allele on the decline in cognition (the incident amnestic MCI or AD group). a Effect of age. Estimated mean trajectory of cognitive decline over time for persons with younger versus older age at enrollment. The dotted line represents a typical subject with 75 th percentile age at enrollment ( 83.8 years), and the solid line refers to a subject with 25 th percentile age at enrollment (74.9 years). b Effect of education. Estimated mean trajectories of cognitive decline over time for groups with lower versus higher level of education. The dotted line represents a typical subject with 75th percentile education (19 years), and the solid line refers to a subject with 25th percentile education (14 years). c Effect of APOE $\varepsilon 4$. Estimated mean trajectories of cognitive decline over time for groups with and without $\varepsilon 4$ allele. The dotted line represents a typical subject without $\varepsilon 4$ allele, and the solid line refers to the one with at least one $\varepsilon 4$ allele.

the location of the change point and rates of decline before and after the change point, providing important clues to where and how risk factors are operating across the full spectrum of cognitive aging.

Using data from two ongoing cohort studies, we compared the location of the estimated change point with the timing of clinical diagnosis. In analyses using data from persons who developed incident $\mathrm{AD}$, we found that on average, more rapid decline in cognitive function started just prior to the time of first clinical diagnosis of $\mathrm{AD}$. Among participants who were cognitively unimpaired at enrollment but later developed amnestic $\mathrm{MCI}$ or $\mathrm{AD}$, lo-
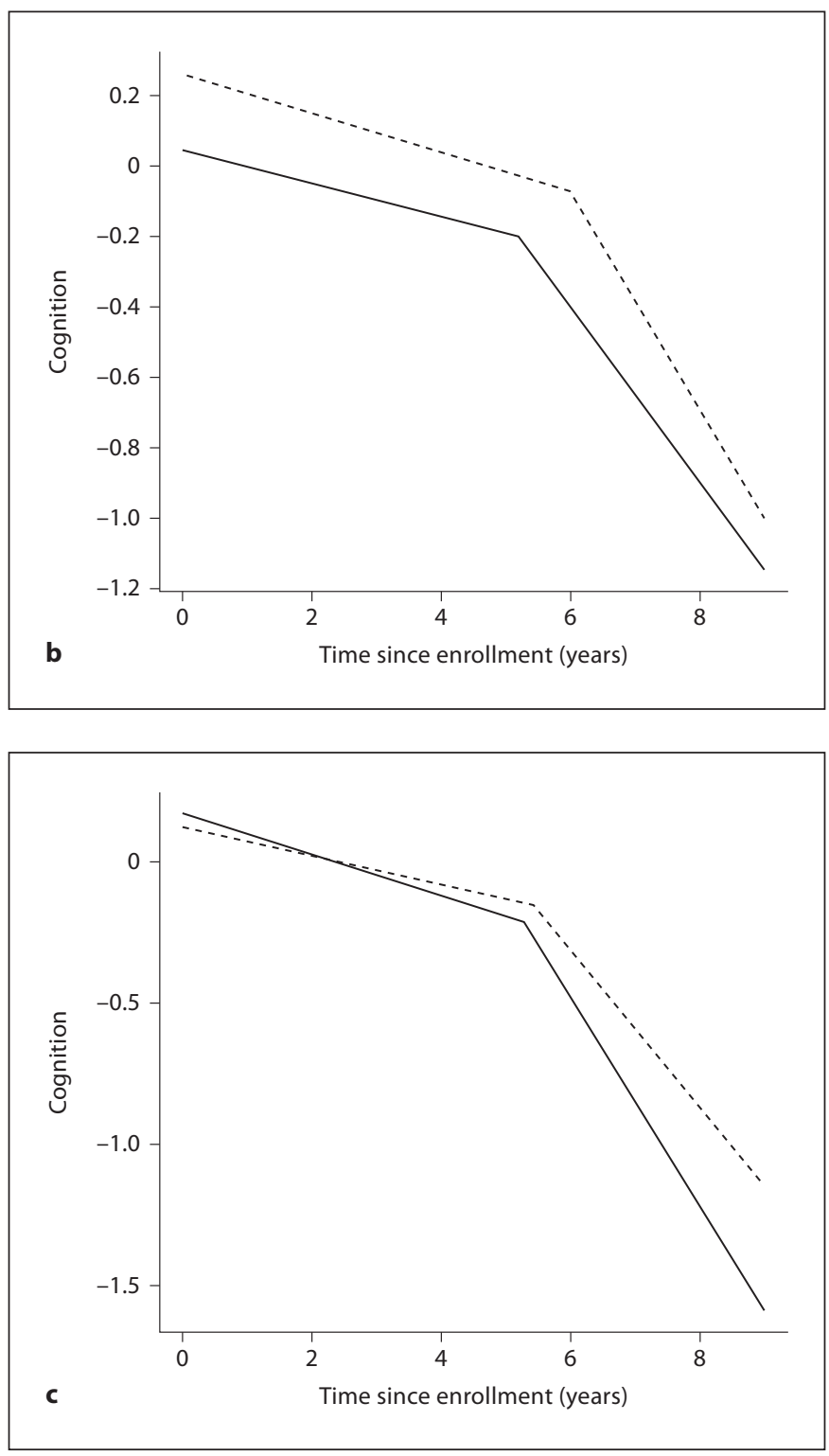

cation of the change point to more rapid decline preceded the diagnosis of clinical AD but followed the diagnosis of amnestic MCI.

We identified several factors that influence the location of the change point and the rates of decline before or after the change point. The older the age at enrollment, the earlier the change point. Age was also associated with the rates of decline before and after the change point among participants who were cognitively unimpaired but later developed amnestic MCI or AD. These results suggest that the association of age with cognitive decline is quite complex. Further, education was associ- 
ated with a delay in the change point and more rapid decline in cognition after the change point. This finding lends analytic support to a conceptual model in which clinical disease is delayed in persons with greater cognitive reserve but people deteriorate more rapidly once disease commences [36]. In terms of $A P O E$ status, we found that $\varepsilon 4$ carriers had more rapid cognitive decline both before and after the change point. Several previous studies have reported that the $\varepsilon 4$ allele was not associated with cognitive decline following the diagnosis of $\mathrm{AD}$ dementia $[22,23]$. We suspect that this is due to prior studies examining this issue among persons with prevalent $\mathrm{AD}$ who already have more severe cognitive impairment. A more recent report found that the $\varepsilon 4$ allele was associated with faster cognitive decline among subjects in the early stages of $\mathrm{AD}$ [37].

Results from our random change point model have broad implications for understanding the relationships between risk factors and cognitive aging. It is likely that risk factors have divergent effects on different phases of the pathophysiological process over the course of AD. AD is the result of a sequence of pathophysiological events from $A \beta$ deposition to synaptic dysfunction, to tangle formation, to other structural changes [11]. Risk factors could be associated with different aspects of this cascade and therefore affect different parts of the cognitive trajectory. Understanding these associations has important implications for the design of clinical trials for diseasemodifying agents.

Several limitations should be noted. Here we restricted the analyses to one change point. It is likely that the trajectory across the continuum of $\mathrm{AD}$ has more than one change point. Unfortunately, more annual repeated measures are needed to explore these potentially more complicated associations. However, as longitudinal cognitive data accrue, we will be able to conduct such analyses in the future. In addition, it is possible to incorporate subjects with fewer repeated measures in the model such that these data contribute partially to the overall longitudinal trajectory. The effect on inference remains unclear, particularly with regard to estimation of the change points. It is an important topic for future studies as it could potentially affect the utility of this modeling approach. Further, the proposed model estimated the random change point relative to the time of study enrollment, as opposed to the time of clinical diagnosis. While using study enrollment as the time reference offers the potential utility of being prospective, the approach might introduce larger variance in estimating the random change point. Future work is necessary to examine how choice of time references changes the inference on trajectory of cognition in the context of random change point models. The study also has many strengths. In particular, it has a large number of persons with incident $\mathrm{AD}$ and incident MCI. Further, cognition is well characterized with 17 cognitive tests administered at evenly spaced annual intervals. Finally, it has an overall follow-up rate over $90 \%$ among survivors, reducing the potential for bias due to differential loss to follow-up.

\section{Acknowledgements}

We are indebted to all the participants of the Religious Order Study and the Rush Memory and Aging Project, as well as the staff at the Rush Alzheimer's Disease Center for this work. This research was supported by National Institute on Aging grants R01AG17917, P30AG10161, R01AG15819, R01AG34374, R01AG33678, R01AG36042, and RC2AG36547.

\section{Appendix A}

In this appendix, we presented the parameter estimates using alternative choices of priors for the data on incident $\mathrm{AD}$ group (model A in table 2, main text) together with the estimates from the main analysis (reference). Two alternative sets of priors were specified below.

Alternative priors 1 still follows the same distribution functions as in the main analysis, but we changed the parameters setup to

$$
\begin{aligned}
& \beta \sim N(10,10,000) \\
& \sum_{4 \times 4}{ }^{-1} \sim \text { Wishart }(R, 10), \text { where } R=I_{4 \times 4}(10) \\
& \frac{1}{\sigma_{e}^{2}} \sim \operatorname{Gamma}(0.1,0.1)
\end{aligned}
$$

Alternative priors 2 has different distribution function for coefficients of fixed effects as well as the residual variance

$$
\begin{aligned}
& \beta \sim \text { uniform }(-10,10) \\
& \sum_{4 \times 4}{ }^{-1} \sim \text { Wishart }(R, 4), \text { where } R=I_{4 \times 4}(0.1) \\
& \sigma_{e} \sim \text { uniform }(0,100)
\end{aligned}
$$




\begin{tabular}{|c|c|c|c|}
\hline Effect & $\begin{array}{l}\text { Reference } \\
\mathrm{EST} \pm \mathrm{SD}\end{array}$ & $\begin{array}{l}\text { Alternative priors } 1 \\
\mathrm{EST} \pm \mathrm{SD}\end{array}$ & $\begin{array}{l}\text { Alternative priors } 2 \\
\mathrm{EST} \pm \mathrm{SD}\end{array}$ \\
\hline Intercept & $-0.076 \pm 0.034$ & $-0.077 \pm 0.036$ & $-0.073 \pm 0.034$ \\
\hline Sex (male) & $-0.147 \pm 0.057$ & $-0.141 \pm 0.061$ & $-0.148 \pm 0.057$ \\
\hline Education, years & $0.042 \pm 0.007$ & $0.041 \pm 0.007$ & $0.042 \pm 0.007$ \\
\hline$A P O E$ & $-0.102 \pm 0.054$ & $-0.099 \pm 0.057$ & $0.102 \pm 0.054$ \\
\hline Sex $\times$ Slope before & $0.006 \pm 0.014$ & $-0.001 \pm 0.029$ & $0.006 \pm 0.014$ \\
\hline Education, years $\times$ Slope before & $0.0003 \pm 0.002$ & $0.001 \pm 0.004$ & $0.0002 \pm 0.002$ \\
\hline$A P O E \times$ Slope before & $-0.033 \pm 0.013$ & $-0.035 \pm 0.027$ & $-0.033 \pm 0.013$ \\
\hline Slope_after & $-0.337 \pm 0.023$ & $-0.351 \pm 0.031$ & $-0.337 \pm 0.023$ \\
\hline Age $\times$ Slope_after & $-0.003 \pm 0.003$ & $-0.002 \pm 0.004$ & $-0.003 \pm 0.003$ \\
\hline Age $\times$ change point & $-0.138 \pm 0.026$ & $-0.133 \pm 0.026$ & $-0.139 \pm 0.026$ \\
\hline Sex $\times$ change point & $-0.608 \pm 0.358$ & $-0.578 \pm 0.356$ & $-0.612 \pm 0.357$ \\
\hline Education, years $\times$ change point & $0.155 \pm 0.045$ & $0.160 \pm 0.046$ & $0.155 \pm 0.045$ \\
\hline$A P O E \times$ change point & $-0.519 \pm 0.337$ & $-0.331 \pm 0.350$ & $-0.518 \pm 0.343$ \\
\hline \multicolumn{4}{|l|}{$\mathrm{SD}$ of random effect } \\
\hline Random intercept & $0.435 \pm 0.129$ & $0.463 \pm 0.134$ & $0.436 \pm 0.129$ \\
\hline Random Slope_before & $0.074 \pm 0.028$ & $0.214 \pm 0.060$ & $0.074 \pm 0.028$ \\
\hline Random Slope_after & $0.247 \pm 0.092$ & $0.370 \pm 0.124$ & $0.247 \pm 0.091$ \\
\hline Random change point & $2.218 \pm 0.716$ & $2.114 \pm 0.690$ & $2.214 \pm 0.719$ \\
\hline
\end{tabular}

Age = Age at enrollment centered at 79; Education, years = years of education centered at 16; $A P O E=1$ for carriers of $\varepsilon 4$ allele, 0 for noncarriers; Slope_before $=$ slope estimate before the change point; Slope_after $=$ slope estimate after the change point.

\section{References}

1 Sloane PD, Zimmerman S, Suchindran C, et al: The public health impact of Alzheimer's disease, 2000-2050: potential implication of treatment advances. Annu Rev Public Health 2002;23:213-231

-2 Brookmeyer R, Gray S, Kawas C: Projections of Alzheimer's disease in the United States and the public health impact of delaying disease onset. Am J Public Health 1998;88: 1337-1342.

-3 Johnson DK, Storandt M, Morris JC, et al: Longitudinal study of the transition from healthy aging to Alzheimer disease. Arch Neurol 2009;66:1254-1259.

4 Grober E, Hall CB, Lipton RB, et al: Memory impairment, executive dysfunction, and intellectual decline in preclinical Alzheimer's disease. J Int Neuropsychol Soc 2008;14: 266-278.

$>5$ Howieson DB, Carlson NE, Moore MM, et al: Trajectory of mild cognitive impairment onset. J Int Neuropsychol Soc 2008;14:192198.
-6 Petersen RC, Parisi JE, Dickson DW, et al: Neuropathologic features of amnestic mild cognitive impairment. Arch Neurol 2006;63: 665-672.

7 Markesbery WR, Schmitt FA, Kryscio RJ, et al: Neuropathologic substrate of mild cognitive impairment. Arch Neurol 2006;63:3846.

$>8$ Bennett DA, Schneider JA, Arvanitakis Z, et al: Neuropathology of older persons without cognitive impairment from two community-based studies. Neurology 2006;66:18371844.

$\checkmark 9$ Albert MS, DeKosky ST, Dickson D, et al: The diagnosis of mild cognitive impairment due to Alzheimer's disease: recommendations from the National Institute on AgingAlzheimer's Association workgroups on diagnostic guidelines for Alzheimer's disease. Alzheimers Dement 2011;7:270-279.
10 McKhann GM, Knopman DS, Chertkow H, et al: The diagnosis of dementia due to Alzheimer's disease: recommendations from the National Institute on Aging-Alzheimer's Association workgroups on diagnostic guidelines for Alzheimer's disease. Alzheimers Dement 2011;7:263-269.

11 Sperling RA, Aisen PS, Beckett LA, et al: Toward defining the preclinical stages of Alzheimer's disease: recommendations from the National Institute on Aging-Alzheimer's Association workgroups on diagnostic guidelines for Alzheimer's disease. Alzheimers Dement 2011;7:280-292.

12 Evans DA, Funkenstein HH, Albert MS, et al: Prevalence of Alzheimer's disease in a community population of older persons: higher than previously reported. JAMA 1989;262:2551-2556.

13 Wilson RS, Beckett LA, Bennett DA, et al: Change in cognitive function in older persons from a community population: relation to age and Alzheimer disease. Arch Neurol 1999;56:1274-1279. 
14 Wilson RS, Li Y, Aggarwal NT, et al: Education and the course of cognitive decline in Alzheimer disease. Neurology 2004;63: 1198-1202.

$\checkmark 15$ Andersen K, Launer LJ, Dewey ME, et al: Gender differences in the incidence of AD and vascular dementia: The EURODEM Studies. EURODEM Incidence Research Group. Neurology 1999;53:1992-1997.

16 Hebert LE, Wilson RS, Gilley DW, et al: Decline of language among women and men with Alzheimer's disease. J Gerontol B Psychol Sci Soc Sci 2000;55:354-360.

- 17 Stern Y, Gurland B, Tatemichi TK, et al: Influence of education and occupation on the incidence of Alzheimer's disease. JAMA 1994;271:1004-1010.

- 18 Stern Y, Albert S, Tang MX, et al: Rate of memory decline in $\mathrm{AD}$ is related to education and occupation: cognitive reserve? Neurology 1999;53:1942-1947.

19 Hall CB, Derby C, LeValley A, et al: Education delays accelerated decline on a memory test in persons who develop dementia. Neurology 2007;69:1657-1664.

20 Schiepers OJ, Harris SE, Gow AJ, et al: APOE E4 status predicts age-related cognitive decline in the ninth decade: longitudinal follow-up of the Lothian Birth Cohort 1921. Mol Psychiatry 2012;17:315-324.

-21 Sando SB, Melquist S, Cannon A, et al: APOE epsilon 4 lowers age at onset and is a high risk factor for Alzheimer's disease; a case control study from central Norway. BMC Neurol 2008;16:8:9.
22 Jonker C, Schmand B, Lindeboom J, Havekes LM, Launer LJ: Association between apolipoprotein E epsilon 4 and the rate of cognitive decline in community-dwelling elderly individuals with and without dementia. Arch Neurol 1998;55:1065-1069.

23 Dal Forno G, Rasmusson DX, Brandt J, Carson KA, Brookmeyer R, Troncoso J, et al: Apolipoprotein $\mathrm{E}$ genotype and rate of decline in probable Alzheimer's disease. Arch Neurol 1996;53:345-350.

24 Bennett DA, Schneider JA, Arvanitakis Z, Wilson RS: Overview and findings from the religious orders study. Curr Alzheimer Res 2011, E-pub ahead of print.

25 Bennett DA, Schneider JA, Buchman AS, Barnes LL, Boyle PA, Wilson RS: Overview and findings from the rush memory and aging project. Curr Alzheimer Res 2012, E-pub ahead of print.

26 Boyle PA, Buchman AS, Wilson RS, et al: The APOE epsilon4 allele is associated with incident mild cognitive impairment among community-dwelling older persons. Neuroepidemiology 2010;34:43-49.

27 Wilson RS, Schneider JA, Boyle PA, Arnold SE, Tang Y, Bennett DA: Chronic distress and incidence of mild cognitive impairment. Neurology 2007;68:2085-2092.

28 Wilson RS, Schneider JA, Barnes LL, Beckett LA, Aggarwal NT, Cochran EJ, et al: The apolipoprotein E epsilon 4 allele and decline in different cognitive systems during a 6-year period. Arch Neurol 2002;59:11541160.

-29 Bennett DA, Schneider JA, Bienias JL, et al: Mild cognitive impairment is related to Alzheimer disease pathology and cerebral infarctions. Neurology 2005;64 834-841.
30 McKhann G, Drachman D, Folstein M, et al: Clinical diagnosis of Alzheimer's disease. Report of the NINCDS-ADRDA Work Group under the auspices of Department of Health and Human Services Task Force on Alzheimer's disease. Neurology 1984;34:939.

31 Bennett DA, Wilson RS, Schneider JA, et al: Natural history of mild cognitive impairment in older persons. Neurology 2002;59: 198-205.

32 Schneider JA, Arvanitakis Z, Leurgans SE, et al: The neuropathology of probable Alzheimer disease and mild cognitive impairment. Ann Neurol 2009;66:200-208.

33 Hall CB, Lipton RB, Sliwinski M, Stewart WF: A change point model for estimating the onset of cognitive decline in preclinical Alzheimer's disease. Stat Med 2000;19:15551566.

34 McArdle JJ, Wang L: Modeling age-based turning points in longitudinal life-span growth curves of cognition; in Cohen P (ed): Applied Data Analytic Techniques for Turning Points Research. New York, Routledge, 2008.

35 Lunn DJ, Thomas A, Best N, Spiegelhalter D: WinBUGS - a Bayesian modelling framework: concepts, structure, and extensibility. Statistics and Computing, 2000;10:325-337.

36 Stern Y: Cognitive reserve and Alzheimer disease. Alzheimer Dis Assoc Disord 2006; 20(3 suppl 2):S69-S74.

>37 Cosentino S, Scarmeas N, Helzner E, Glymour MM, Brandt J, Albert M, et al: APOE epsilon 4 allele predicts faster cognitive decline in mild Alzheimer disease. Neurology 2008;70(19 Pt 2):1842-1849. 